\title{
Standard-Model Extension Constraints on Lorentz and CPT Violation From Optical Polarimetry of Active Galactic Nuclei
}

\author{
Andrew S. Friedman, ${ }^{1}$ David Leon, ${ }^{1}$ Roman Gerasimov,${ }^{1}$ Kevin D. Crowley,${ }^{1}$ Isaac \\ Broudy, ${ }^{1}$ Yash Melkani, ${ }^{1}$ Walker Stevens, ${ }^{1}$ Delwin Johnson,,${ }^{1}$ Grant Teply,${ }^{1}$ David \\ Tytler, ${ }^{1}$ Brian G. Keating, ${ }^{1}$ and Gary M. Cole ${ }^{2}$ \\ ${ }^{1}$ Center for Astrophysics and Space Sciences, University of California, \\ San Diego, La Jolla, CA 92093, USA \\ ${ }^{2}$ Western Nevada College, Carson City, NV 89703, USA
}

\begin{abstract}
Vacuum birefringence from Lorentz and CPT violation in the Standard-Model Extension can be constrained using ground-based optical polarimetry of extragalactic sources. We describe results from a pilot program with an automated system that can perform simultaneous optical polarimetry in multiple passbands on different telescopes with an effective $0.45 \mathrm{~m}$ aperture. ${ }^{1}$ Despite the limited collecting area, our polarization measurements of AGN using a wider effective optical passband than previous studies yielded individual line-of-sight constraints for Standard-Model Extension mass dimension $d=5$ operators within a factor of about one to ten of comparable broadband polarimetric bounds obtained using data from a $3.6 \mathrm{~m}$ telescope with roughly 64 times the collecting area. ${ }^{2}$ Constraining more general anisotropic Standard-Model Extension coefficients at higher $d$ would require more AGN along different lines of sight. This motivates a future dedicated ground-based, multi-band, optical polarimetry AGN survey with $\gtrsim 1 \mathrm{~m}$-class telescopes, to obtain state-of-the-art anisotropic Standard-Model Extension $d=4,5,6$ constraints, while also using complementary archival polarimetry. This could happen more quickly and costeffectively than via spectropolarimetry and long before more competitive constraints from space- or balloon-based x-ray $/ \gamma$-ray polarization measurements.
\end{abstract}

The Standard-Model Extension (SME) ${ }^{3}$ allows for the violation of both Lorentz and CPT symmetry. Some SME coefficients predict vacuum birefringence, resulting in a wavelength-dependent rotation of the plane of linear polarization for photons. Such SME effects would increasingly depolarize light traveling over cosmological distances, with stronger observable effects predicted at higher redshifts and higher energies. Broadband polarimetry and spectropolarimetry of high-redshift extragalactic sources can thus be used to place increasingly sensitive astrophysical bounds on the SME. 
Since SME effects can vary across the sky, one requires multiple measurements along different lines of sight to adequately constrain the most natural anisotropic SME models: the number $N(d)$ of distinct anisotropic vacuum-birefringent SME coefficients increases according to $N(d)=(d-1)^{2}$ and $N(d)=2(d-1)^{2}-8$ for odd and even mass dimension $d$, respectively. Thus, the mass dimensions $d=5,7,9, \ldots$ and $d=4,6,8,10, \ldots$, require respective polarization measurements along at least $N(d)=16,36,64, \ldots$ or $10,42,90,154, \ldots$ independent lines of sight for full coefficient coverage.

Space- or balloon-based $\mathrm{x}$-ray $/ \gamma$-ray polarimetry can yield very sensitive line-of-sight constraints on the SME. ${ }^{4}$ However, there are currently only about ten published $\mathrm{x}$-ray $/ \gamma$-ray polarization measurements of gamma-ray bursts (GRBs) that are not upper/lower limits. ${ }^{4,5}$ By contrast, there are thousands of AGN with published broadband optical polarimetry and hundreds with spectropolarimetry. ${ }^{7,8}$ At present, it is thus much more feasible to obtain wider sky coverage quickly, including many sources over a range of redshifts, by analyzing archival polarimetry of the most highly polarized AGN, including BL Lacs, Blazars, and highly polarized quasars.

Ultimately, advances in space- or balloon-based x-ray $/ \gamma$-ray polarimetry of high-redshift transient GRBs could provide significantly stronger bounds on anisotropic SME coefficients than optical AGN polarimetry in the coming decades. ${ }^{2,4,6,9}$ However, since ground-based optical polarimetry and spectropolarimetry have smaller statistical and systematic errors than the more expensive and difficult x-ray $/ \gamma$-ray polarimetry measurements, optical studies of AGN - the brightest, continuous, highly polarized, extragalactic optical sources - represent the most cost-effective approach to improve constraints on anisotropic SME coefficients today. ${ }^{1,2,10}$

One can test CPT-odd birefringent SME coefficients with broadband polarimetry as follows. ${ }^{1-3}$ The $k_{(V) j m}^{(d)}$ SME coefficients predict a rotation of the linear polarization plane. For two photons with energies $E_{1}<E_{2}$ emitted in the rest frame of a source at redshift $z$ with the same polarization, the difference in polarization angle observed on Earth is

$$
\Delta \psi^{(d)}(z) \approx\left(E_{2}^{d-3}-E_{1}^{d-3}\right) L^{(d)}(z) \sum_{j m} Y_{j m}(\theta, \phi) k_{(V) j m}^{(d)},
$$

where $Y_{j m}(\theta, \phi)$ are the spin-weighted spherical harmonics with celestial coordinates $(\theta, \phi)$, and $L^{(d)}(z)=\int_{0}^{z} \frac{\left(1+z^{\prime}\right)^{d-4}}{H\left(z^{\prime}\right)} d z^{\prime}$ is the effective comoving distance; $H(z)$ is the Hubble parameter for a FRW cosmology.

If we conservatively assume $100 \%$ intrinsic source polarization at all wavelengths, ${ }^{1,2,10}$ integrating Eq. (1) over the effective energy bandpass 
$T(E)$ yields intensity-normalized Stokes parameters $q^{(d)}(z)$ and $u^{(d)}(z)$ via

$$
q^{(d)}(z)+i u^{(d)}(z)=\int_{E_{1}}^{E_{2}} \exp \left[i\left(E^{d-3}-E_{1}^{d-3}\right) \xi(z)\right] T(E) d E
$$

where $\xi(z) \equiv 2 L^{(d)}(z) \bar{k}_{(V)}^{(d)}$ and $\bar{k}_{(V)}^{(d)} \equiv \sum_{j m} Y_{j m}(\theta, \phi) k_{(V) j m}^{(d)}$.

The polarization $p_{\max }^{(d)}(z)=\left(\left[q^{(d)}(z)\right]^{2}+\left[u^{(d)}(z)\right]^{2}\right)^{1 / 2}$ represents the theoretical maximum observable in the SME. Measuring a polarization fraction $p_{\star} \pm n \sigma_{p_{\star}}<p_{\max }^{(d)}(z)$ can thus directly yield an $n-\sigma$ upper bound on the coefficient combination $\bar{k}_{(V)}^{(d)}$. A spherical-harmonic decomposition on the sky can then be used to combine sufficient numbers of line-of-sight constraints to bound all $N(d)$ parameters at a given $d .{ }^{2,10}$ These can also include lineof-sight constraints from spectropolarimetry, which can be about two or three orders of magnitude more sensitive than broadband polarimetry for $d=5{ }^{2}$ Tests of CPT-even $d=4$ SME coefficients are discussed in Ref. 10.

The Array Photo Polarimeter (APPOL) is a pilot program with an automated small telescope system. ${ }^{1}$ It can conduct high-cadence, faintobject, optical polarimetry in multiple passbands with polarization-fraction statistical errors $\sigma_{p} \lesssim 0.5-1 \%$ for targets with visual band magnitude $V \lesssim$ 14-15 mag, and systematic errors $\sigma_{p} \sim 0.04 \%$. These are competitive with the best ground-based optical-telescope measurements. APPOL is located at StarPhysics Observatory in Reno, Nevada at an elevation of $1585 \mathrm{~m}$ and serves as a test bed for future polarimeters that could be installed on $\gtrsim 1 \mathrm{~m}$ class telescopes capable of observing much fainter AGN. ${ }^{1}$

APPOL uses dual-beam inversion optical polarimetry with Savart plate analyzers rotated through a half-wave-plate image sequence; ${ }^{11}$ it employs an automated telescope, filter, and instrument-control system with five colocated telescopes on two mounts. We combined simultaneous polarimetry from two co-located Celestron 11- and 14-inch telescopes with an effective 17.8 inch $(0.45 \mathrm{~m})$ telescope diameter with $\mathrm{Lum}$ and $I_{c}$ filters into an effective optical bandpass with high transmission over the $\lambda \simeq 400-900 \mathrm{~nm}$ range of the two filters. ${ }^{1}$ This yields more stringent SME bounds than either filter alone and achieves the effective collecting power of a larger telescope.

Our initial APPOL campaign observed two sources: BL Lacertae and S5 B0716 +714 at redshifts $z=0.069$ and $z=0.31$, respectively. This can only give SME line-of-sight constraints or bound the $k_{(V) 00}^{(d)}$ isotropic CPTodd SME coefficient. ${ }^{1}$ Simultaneous optical polarimetry with our Lum $+I_{c}$ filter can yield SME line-of-sight constraints that are theoretically up to ten times $(d=5)$ and 30 times $(d=6)$ more sensitive than in the $I_{c}$ band alone. Despite our small effective $0.45 \mathrm{~m}$ aperture, we achieved $d=5$ line-of-sight 
constraints within a factor of up to ten in sensitivity compared to relevant constraints using broadband optical polarimetry with a $V$-band filter on a $3.6 \mathrm{~m}$ telescope with roughly 64 times the collecting area. ${ }^{1,2}$

Using archival optical polarimetry and spectropolarimetry for AGN (and GRB afterglows), there is a unique opportunity to test $k_{(V)}^{(d)} \mathrm{SME}$ coefficients about one to two orders of magnitude better than previous work. ${ }^{2,10}$ While most archival optical data used a single filter, we conjecture that simultaneous observations in as few as two filters could constrain $k_{(V)}^{(d)}$ more cost-effectively than spectropolarimetry on $\gtrsim 2 \mathrm{~m}$-class telescopes. We are testing this now by performing simultaneous two-band optical polarimetry on roughly 10-20 of the brightest, highly polarized, AGN that an upgraded $0.5 \mathrm{~m}$ APPOL system can reasonably observe. This will enable design-feasibility studies for a ground-based, multi-band, optical polarimetry survey of high-redshift AGN with $\gtrsim 1 \mathrm{~m}$-class telescopes. Finally, with thousands of sources over the sky, archival and new optical polarimetry could, for the first time, provide sufficient data to constrain not just individual $k_{(V)}^{(d)}$, but also a possible redshift dependence of any SME coefficients, potentially revealing time variation of the underlying fields and elucidating the role of the associated new physics over cosmic history.

\section{Acknowledgments}

ASF and BGK acknowledge support from NSF INSPIRE Award PHYS 1541160 and UCSD's Ax Center for Experimental Cosmology. DT is supported in part by NSF award AST1413568.

\section{References}

1. A.S. Friedman et al., Phys. Rev. D 99, 035035 (2019).

2. F. Kislat and H. Krawczynski, Phys. Rev. D 95, 083013 (2017).

3. V.A. Kostelecký and M. Mewes, Phys. Rev. D 80, 015020 (2009).

4. V.A. Kostelecký and M. Mewes, Phys. Rev. Lett. 110, 201601 (2013).

5. J. Wei, Mon. Not. R. Astron. Soc. 485, 2401 (2019).

6. M. Pearce et al., Astropart. Phys. 104, 54 (2019).

7. D. Sluse et al., Astron. Astrophys. 433, 757 (2005).

8. P.S. Smith et al., in N. Johnson and D. Thompson, eds., Proceedings of the 2009 Fermi Symposium, eConf C0911022, 2009.

9. F. Kislat, talk 109.79 presented at 17th HEAD Meeting of the American Astronomical Society, Monterey, 17-21 March, 2019.

10. F. Kislat, Symmetry 10, 596 (2018).

11. J. Tinbergen, Astronomical Polarimetry, Cambridge University Press, Cambridge, 2005. 